\title{
ЛИТЕРАТУРНЫЙ ОБЗОР
}

УДК: 618.1-089; 617-089.844

\section{РЕЦИДИВНОЕ НЕДЕРЖАНИЕ МОЧИ}

Касян Г.Р., Гвоздев М.Ю., Куприянов Ю.А., Глотов А В., Строганов Р.В. Кафедра урологии, ГБОУ ВПО Московский государственный медикостоматологический университет Минздрава России, г.Москва

Адрес: 125206, г.Москва, ул. Вучетича, 21, корпус 3, тел. (495)6113129

Эл.почта: g.kasyan@gmail.com, gvozdev@mail.ru,dr.kupriyanov@mail.ru, r.v.stroganov@gmail.com

Проведен анализ данных зарубежной литературы в отнотении лечения рециидвов недержания мочи у женщин. Стандартов лечения рецидивной формы недержания мочи на сегодняшний день не существует. Эффективность повторных оперативных вмешательств несколько ниже первичных. Эффективность повторных петлевых пластик, выполненных из позадилонного доступа выше по сравнению с трансобтураторным. Наиболее часто и эффективно применялась операция TVT. Детальное обследование пачиенток помогает повысить эффективность проводимого лечения.

Ключевые слова: рецидивное недержание мочи; субуретральная петлевая пластика; TVT; TOT

\section{RECURRENT URINARY INCONTINENCE}

\section{Kasyan G.R., Gvozdev M.Y., Kupriyanov Y.A., Glotov A.V., Stroganov R.V.}

Department of Urology Moscow State University Of Medicine And Dentistry, Moscow

The analysis of the data of foreign literature in the treatment of recurrence of urinary incontinence in women. Standards for treatment of recurrent forms of urinary incontinence to date does not exist. Efficiency reoperation slightly lower primary. Efficacy of repeat loop plastics made from retropubic access higher than transobturator. The most commonly used and effective operation TVT. A detailed survey of patients helps to increase the effectiveness of the treatment.

Key words: recurrent urinary incontinence; suburethral sling procedure; TVT; TOT

\section{ВВЕДЕНИЕ}

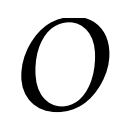

дной из наиболее обсуждаемых проблем в женской урологии на сегодняшний день является недержание мочи при напряжении. В связи с существенным снижением качества жизни, немаловажное значение отводится хирургическим методам лечения данного заболевания. Основным методом лечения являются субуретральные петлевые пластики с использованием синтетических протезов. По данным зарубежной литературы последние показывают высокую эффективность (90\%), а также отмечается рост числа прово- димых операций. При условии, что эффективность данных операций не будет снижаться, количество пациенток, у которых операция окажется неэффективной остается значительным. На сегодняшний день четких рекомендаций по выбору тактики лечения больных с рецидивом недержания мочи нет. К методам лечения данной формы заболевания относятся - операция Burch, инъекции объемобразующих препаратов, имплантации искусственного сфинктера мочевого пузыря, различные варианты коррекции ранее выполненной петлевой пластики, повторная операция и 
применение регулируемых слингов. Значительная часть публикаций, посвященных лечению рецидива недержания мочи у женщин сводится к оценке эффективности субуретральной петлевой пластики и проведению повторных хирургических вмешательств.

Целью данной работы является анализ литературы, посвященной развитию и методам оперативного лечения рецидивного недержания мочи.

\section{МАТЕРИАЛЫ И МЕТОДЫ}

Нами выполнен поиск литературы с использованием поисковых систем: www.ncbi.nlm.nih.gov/pubmed и http:// www.thecochranelibrary.com.

Эффективность субуретральных петлевых операций остается не до конца изученной, в частности, Van Baelen [1] приводит результаты исследования 19 пациенток, которым после различных субуретральных петлевых пластик выполнялась операция ТОТ, ее эффективность составила 55\%. В исследовании Azam [2] 67 пациенткам с рецидивом недержания мочи выполнялась операция TVT, ее эффективность составила $81 \%$, при этом перфорация мочевого пузыря при проведении троакаров отмечена у 13 пациенток.

В опубликованной работе Lo [3] , 41 больной с рецидивом недержания мочи была выполнена операция TVT. Успех операции отмечен у 82,9\%, улучшение отмечено у 4,9\% и у 12,2\% положительный эффект не отмечен.
Ряд авторов под руководством AbdelFattah [4] публикуют результаты работы, в которой 46 пациенткам с рецидивом недержания мочи выполнялись операция TVT-O или операция TOT. Эффективность оценивалась спустя год, составила 69,6\% и 76,5\%.

В исследовании Stav [5] в которое были включены 1225 больных, 955 пациенткам была выполнена петлевая пластика из позадилонного доступа, 270 - из трансобтураторного. 77 из них выполнялась повторно. Успех достигнут в $62 \%$ случаев при повторной операции и $86 \%$ в группе первичной операции. Имплантация позадилонной петли (71\%) чаще приводила к успешному исходу, нежели траносбтураторная петля (48\%).

По результатам исследования Le [6], в котором 13 пациенткам с рецидивным недержанием мочи петля была имплантирована из позадилонного доступа, а 16 пациенткам из трансобтураторного, большую эффективность показала первая (92,3\% против 62,5\%).

Группа ученых во главе с Rardin [7] приводит результаты исследования, в котором 245 пациенткам была выполнена операция TVT. У 87 из них различные оперативные вмешательства по поводу недержания мочи были в анамнезе. Эффективность первично и вторично выполненных операций была приблизительно одинаковой $-87 \%$ и $85 \%$.

Другие авторы (Ala-Nissila [8]) представляют результаты исследования, в 
котором 60 больным с рецидивным недержанием мочи выполнялась петлевая пластика с использованием TVT. Операция показала положительный эффект в $85 \%$ случаев.

Группа авторов (Sivaslioglu et al. [9]) опубликовали данные исследования, в котором 29 пациенткам с рецидивным недержанием мочи, перенесшим раннее операцию Burch выполнялись операции TVT (23 пациентки) и TOT (6 пациенток). Результаты следующие: операция TVT показала эффективность в $62,1 \%$ случаев, операция ТОТ - в 66,6\%.

В исследовании De Ridder [10], включающем 80 пациенток, перенесших различные петлевые операции, представлены следующие результаты - 26 больным (33\%) была выполнена операция TOT, 25 (31\%) - операция TVT, 16 пациенткам (20\%) был имплантирован мини-слинг и 13 пациенток (15\%) перенесли имплантацию петли из свиной кожи (Pelvicol). 4 пациенткам (5\%) выполнялось рассечение ранее установленной петли. В 6 случаях (7,5\%) выполнялось ее удаление. Суммарная эффективность всех операций составила 63,5\%. Субъективно довольны результатом операции 74\% пациенток. При этом значимых различий в эффективности операции между петлями, за исключением петли Pelvicol, обнаружено не было.

Доктор Liapis [11] сообщает о применении операции TVT у больных с рецидивом недержания мочи после петлевых операций. В исследование было включена 31 пациентка. Срок наблюдения составил 18 месяцев. Операция была успешна в 74\% случаев (потеря менее 1 г мочи в течение одночасового теста с прокладкой), еще в $6.5 \%$ отмечалось улучшение состояния.

В обширном систематическом обзоре Pradhan [12] привел следующие результаты по поводу петлевых пластик: успех операции при стрессовом недержании составляет 78,5\%, успех повторной петлевой пластики - 73,3\%. Конкретизируя статистические данные успех операции TVT составляет 79,8\%, операции ТОТ - 54\%. В 28 случаях из 363 проведенных имплантаций позадилонной петли отмечены случаи перфорации мочевого пузыря. Latthe [13] в своих исследованиях приводит пример травмы мочевыделительной системы у 10 пациенток из 238 в результате аналогичных операций

Необходимо отметить, что опыта применения инъекций объемобразующих препаратов в лечении рецидивной формы недержания мочи практически нет. По разным данным эффективность данного метода лечения при первичном недержании мочи при напряжении составляет около $60-70 \%$, и в дальнейшем эффект снижается до $30 \%$, что требует его повторного введения. Lee [14] в своем исследовании изучал эффективность периуретральных инъекций у пациенток с рецидивом недержания мочи после петлевой операции. 
23 пациенткам проводились инъекции полидиметилксилоксана и покрытого карбоном циркония. 34,8\% пациенток полностью удерживали мочу, 77\% отмечали улучшение своего состояния. Под руководством Villet [15] опубликована работа, посвященная увеличению степени натяжения ранее установленной петли. У пациентки, перенесшей операцию TVT, наблюдался рецидив недержания мочи. При обследовании была установлено, что натяжение петли полностью отсутствовало. Villet [15] наложил на ее среднюю часть несколько швов, использую пролен 4/0. В результате этой манипуляции натяжение петли возрасло, что позволило пациентке удерживать мочу. В дальнейшем появилось несколько работ, в которых предлагались разные варианты укорочения и подтягивания ранее имплантируемой петли - накладывание 8-образных швов, непрерывного шва и клипов. Эффективность указанных методов варьировала от 43\% до 72\% [16].

Исходя из анализа публикаций, касающихся оперативного способа лечения рецидивного недержания мочи, можно сделать вывод, что единого мнения в решении этой проблемы нет. Можно предположить, что создание единой базы данных по повторным оперативным вмешательствам при недержании мочи значительно облегчит выбор тактики лечения и создаст доказательную основу, которая поможет учесть максимальное количество кри- териев для индивидуального подхода к каждой больной.

\section{ОБСУЖДЕНИЕ}

Приоритетной задачей в выборе тактики лечения данной группы пациенток является определение причины развития рецидива. В связи с тем, что в настоящее время в большинстве случаев при первичном недержании мочи выполняются субуретральные петлевые пластики, основной причиной может являться расположение протеза, не позволяющее ему полноценно выполнять его функцию.

Немаловажное значение имеет тщательное обследование данной группы пациенток. Оценка факторов риска, таких как возраст, ожирение, наличие гиперактивности мочевого пузыря, сопутствующее оперативное лечение пролапca гениталий, использование общей анестезии, малый опыт хирурга, высокие значения Qmax и объема теряемой мочи, а так же состояние внутреннего сфинктера уретры и ее гипермобильность, помогает повысить эффективность планируемого лечения.

При выборе тактики лечения необходимо полноценное обследование, включающее осмотр, цистоскопию, комплексное уродинамическое исследование. Следует отметить, что проведение УЗИ, МРТ позволяет визуализировать раннее установленный синтетический протез. Flock [17] проводил исследования позиции петли с помощью УЗИ, 
оценивая также ее расположение к общей длине уретры и к ее гипоэхогенному центру. У пациенток, перенесших постановку TVT и решивших проблему недержания, соотношение места расположения петли к общей длине мочеиспускательного канала составило 61\%, в свою очередь расстояние до гипоэхогенного центра в среднем 3,5 мм. Похожие результаты приводит в своих исследованиях Rinne [18] применяя тех- нологию МРТ. В отличие от пациенток с благополучным исходом постановки петли, у пациенток с рецидивом (около $32 \%)$ расположение петли было в проксимальной или дистальной части уретры. Таким образом, основными вопросами для хирурга являются определение сроков проведения повторной операции, целесообразность удаления или коррекции раннее установленного протеза.

\section{ЛИТЕРАТУРА}

1. Van Baelen, A.A. Repeat transobturator tape after failed mid-urethral sling procedure: follow-up with questionnaire-based assessment. // A.A. Van Baelen, K.P. Delaere // Urol Int - Vol.83(4). - P.399-403.

2 . The tension-free vaginal tape procedure in women with previous failed stress incontinence surgery. / U. Azam, M.I. Frazer, E.L. Kozman et al. // J Urol - Vol.166(2). - P.554-556.

3. Tension-free vaginal tape procedure after previous failure in incontinence surgery. / T.S. Lo, S.G. Horng, C.L. Chang et al. // Urology 60(1):57-61

4. Abdel-Fattah, M.F. Single-incision mini-Slings versus Standard midurethral Slings in Surgical management of Female Stress Urinary incontinence: a metaanalysis of effectiveness and complications. / M.F. Abdel-Fattah; C.P. Lim; P. Madhuvrata // European Urology. 2011. - Vol.60. - P.468-480.
5. Long-term outcomes of patients who failed to attend following midurethral sling surgery - a comparative study and analysis of risk factors for non-attendance. / K. Stav et al.,// Aust N Z J Obstet Gynaecol. 2010. - Vol.50(2). - P.173-178.

6. Outcomes following repeat mid urethral synthetic sling after failure of the initial sling procedure: rediscovery of the tensionfree vaginal tape procedure. / K.S. Lee et al. // J Urol. - 2007. - Vol.178 - P.1370-1374.

7. Tension-free vaginal tape: outcomes among women with primary versus recurrent stress urinary incontinence. / C.R. Rardin, N. Kohli, P.L. Rosenblatt et al. // Obstet Gynecol. - 2002. - Nov, Vol.100(5 Pt 1). P.893-897.

8. Tension-free vaginal tape - a suitable procedure for patients with recurrent stress urinary incontinence. / S. Ala-Nissilä, M. Haarala, J. Mäkinen // Acta Obstet Gynecol Scand. - Vol.89(2). - P.210-216. 
9. The management of recurrent cases after the Burch colposuspension: 7 years experience. / A.A. Sivaslioglu, E. Unlubilgin, H.L. Keskin // Arch Gynecol Obstet - Vol.283(4). - P.787-790.

10. De Ridder, D. A repeat midurethral sling as valuable treatment for persistent or recurrent stress urinary incontinence. / D. De Ridder, F. Van der Aa // Int Urogynecol J. - 2013. - Jun, Vol.24(6). - P.:999-1004. Epub. - 2012. - Oct, 24.

11. Liapis, A. Tension-free vaginal tape in the management of recurrent urodynamic stress incontinence after previous failed midurethral tape. / A. Liapis, P. Bakas, G. Creatsas // Eur Urol - 2009. - Vol.55(6). - P.1450-1455.

12. Pradhan, A. Effectiveness of midurethral slings in recurrent stress urinary incontinence: a systematic review and meta-analysis. / A. Pradhan, P. Jain, P.M. Latthe // Int Urogynecol J. - 2012. Jul, Vol.23(7). - P.831-841.

13. Pradhan, A. Effectiveness of midurethral slings in recurrent stress urinary incontinence: a systematic review and meta-analysis. / A. Pradhan, P. Jain, P.M. Latthe // Int Urogynecol J. - 2012. - Jul, Vol.23(7). - P.831-841.
14. Outcomes following repeat mid urethral synthetic sling after failure of the initial sling procedure: rediscovery of the tension-free vaginal tape procedure. / K.S. Lee et al. // J Urol - 2007. - Vol.178. - P.1370-1374.

15. Second tension-free vaginal tape procedure and mesh retensioning: two possibilities of treatment of recurrentpersistent genuine stress urinary incontinence after a primary tensionfree vaginal tape procedure. / R. Villet, A. Ercoli, D. Atallah et al. // Int Urogynecol J Pelvic Floor Dysfunct - 2002. - Vol.13. Vol.377-379.

16. Treatment for unsuccessful tensionfree vaginal tape operation by shortening pre-implanted tape. / T.S. Lo, A.C. Wang, C.C. Liang et al. // J Urol - 2006. Vol.175. - P.2196-2199.

17. Ultrasound assessment of tensionfree vaginal tape (TVT). / F. Flock, F. Kohorst, R. Kreienberg et al. // Ultraschall Med. - 2011. - Jan, Vol.32. - P.35-40.

18. Dynamic MRI confirms support of the mid-urethra by TVT and TVT-O surgery for stress incontinence. / K. Rinne, S. Kainulainen, S. Aukee et al. // Acta Obstet Gynecol Scand. - 2011. - Jun, Vol.90(6). - P.629-635. 